\title{
Prevalence and impact of diabetes among people infected with SARS-CoV-2
}

\author{
G. P. Fadini ${ }^{1}$ M. L. Morieri ${ }^{1}$ E. Longato ${ }^{2} \cdot$ A. Avogaro $^{1}$
}

Received: 15 March 2020 / Accepted: 20 March 2020 / Published online: 28 March 2020

(c) Italian Society of Endocrinology (SIE) 2020

\section{To the Editor}

In December 2019, a new coronavirus causing severe acute respiratory syndrome (SARS-CoV-2) emerged in Wuhan, China. The virus spread rapidly to more than 150 countries and, by the time we are writing (March 19th 2020), it affected $>230,000$ individuals causing almost 10,000 deaths [1].

It has been suggested that diabetes mellitus is one of the most common comorbidities in infected people, but its exact prevalence is unclear. We show results of a meta-analysis of studies reporting the prevalence of diabetes among people infected with the SARS-CoV-2 and its impact on disease severity or progression. We retrieved 12 studies reporting data from 2108 Chinese patients with confirmed SARSCov-2 infection (Data supplement). Mean age was 49.6 years and the prevalence of diabetes was $10.3 \%$ (Fig. 1a). For comparison, the nationwide prevalence of diabetes in China in 2013 was $10.9 \%$ overall and $12.3 \%$ among people aged 40-59 [2].

As of March 19th 2020, Italy was the second country most affected with SARS-Cov-2 $(n=41,035$ individuals with confirmed SARS-CoV-2). At the University Hospital of Padova, located at the centre of an outbreak, among 146 hospitalized patients with confirmed SARS-CoV-2 infection and a mean age of 65.3 years, 13 had pre-existing diabetes, yielding a prevalence of $8.9 \%$ (95\% CI 5.3-14.6). For comparison, the prevalence of diabetes in the same Region

Electronic supplementary material The online version of this article (https://doi.org/10.1007/s40618-020-01236-2) contains supplementary material, which is available to authorized users.

G. P. Fadini

gianpaolo.fadini@unipd.it

1 Department of Medicine, University of Padova, Via Giustiniani 2, 35128 Padua, Italy

2 Department of Information Engineering, University of Padova, Padua, Italy in 2018 was $6.2 \%$ overall and $11.0 \%$ among people aged 55-75 years (mean 65) [3]. A relatively low prevalence of diabetes among SARS-CoV-2 infected people could be due to under-reporting, chance, or a biological phenomenon. Dipeptidyl peptidase-4 (DPP-4) was discovered as a receptor for middle-east respiratory syndrome coronavirus while SARS-CoV-2 appears to use preferentially angiotensin-converting enzyme 2 to enter the cell [4]. However, since DPP-4 inhibitors are popular glucose-lowering medications worldwide, it will be of interest to explore whether they might protect against SARS-CoV-2 infection.

Six of the meta-analysed studies on Chinese patients reported the prevalence of diabetes according to disease severity or outcome ( $n=1687$ patients). The pooled rate ratio of diabetes among patients with adverse disease course as compared to those with the more favourable course was 2.26 (95\% CI 1.47-3.49) (Fig. 1b and Supplemental Table 1).

As of March 17th, the median age of 2003 Italian patients who died while being infected with SARS-CoV-2 was 80.5 (IQR 31-103) and 70\% were men. Among 355 deceased patients with available information on comorbidities, diabetes prevalence was $35.5 \%$ [5]. In 2018, diabetes prevalence among Italian citizens with the same age range and sex distribution was $20.3 \%$ [6]. Thus, the rate ratio of diabetes among patients who died with SARS-CoV-2 infection compared to the general population was 1.75 .

Based on these data, we conclude that diabetes may not increase the risk of SARS-CoV-2 infection but can worsen the outcome of this new coronavirus disease. This finding is consistent with the association between diabetes and excess mortality from any acute and chronic condition, including infections [7]. The situation is rapidly evolving, and close monitoring of these data are important for a correct communication to patients and healthcare providers. 
Fig. 1 a Forest plot of diabetes prevalence among SARS-CoV-2 infected patients. b Forest plot of diabetes rate ratio (RR) among patients with more severe versus those with less severe infection
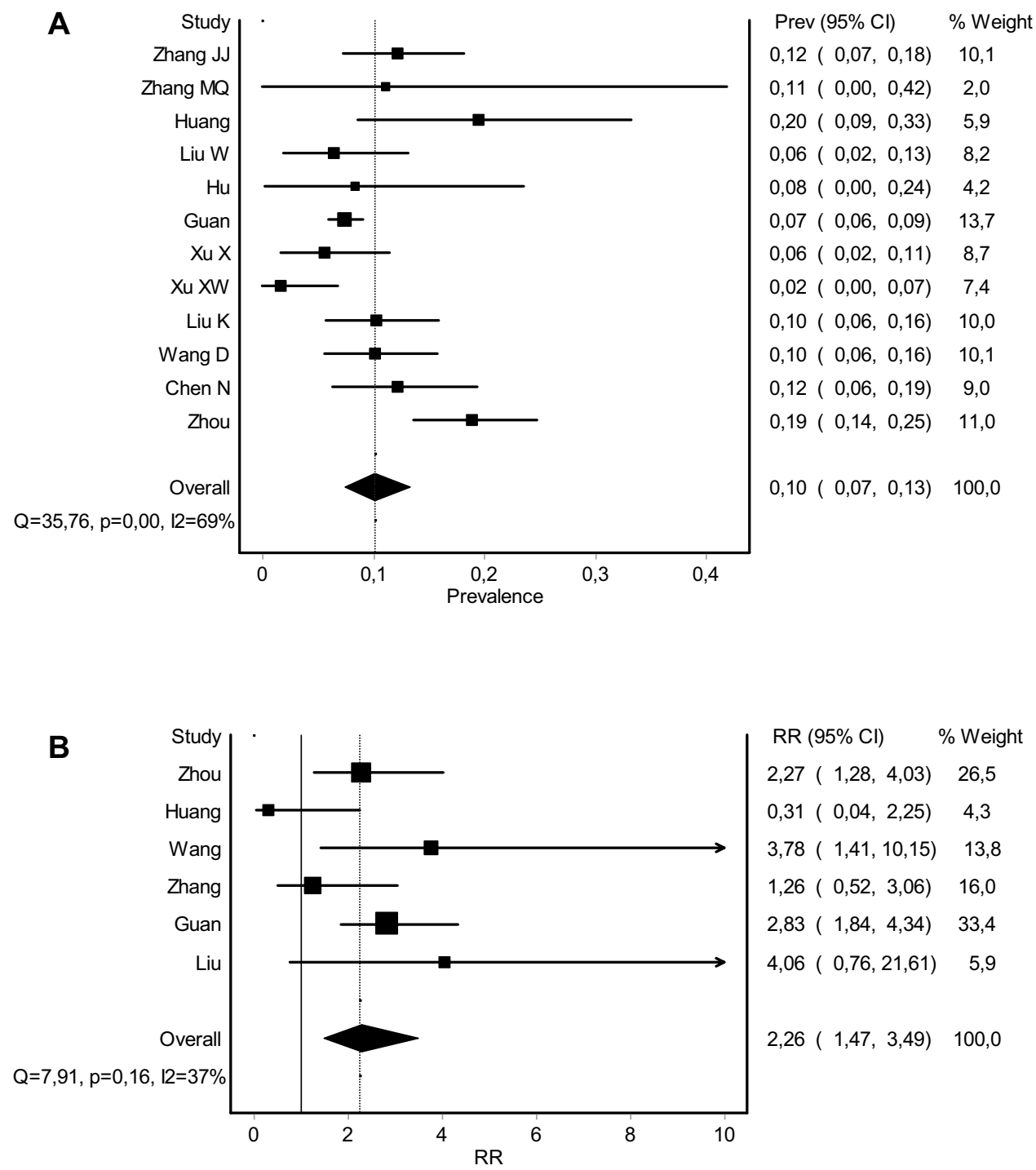

Funding None.

\section{Compliance with ethical standards}

Conflict of interest The authors declare that they have no competing interest.

Ethical approval The study has been conducted in compliance with ethical standards for research involving human participants. Protocol approval for evaluation of the healthcare status in diabetic patients was obtained from the Ethical Committee of the University Hospital of Padova.

Informed consent No informed consent was collected for this study because we used aggregated data or data that are publicly available.

\section{References}

1. John Hopkins University of Medicine: Coronavirus Resource Center. https://coronavirus.jhu.edu/map.html. Accessed 19 Mar 2020
2. Wang L, Gao P, Zhang M, Huang Z, Zhang D, Deng Q, Li Y, Zhao Z, Qin X, Jin D, Zhou M, Tang X, Hu Y (2017) Prevalence and ethnic pattern of diabetes and prediabetes in China in 2013. JAMA 317:2515-2523

3. Longato E, Di Camillo B, Sparacino G, Saccavini C, Avogaro A, Fadini GP (2020) Diabetes diagnosis from administrative claims and estimation of the true prevalence of diabetes among 4.2 million individuals of the Veneto region (North East Italy). Nutr Metab Cardiovasc Dis 30:84-91

4. Letko M, Marzi A, Munster V (2020) Functional assessment of cell entry and receptor usage for SARS-CoV-2 and other lineage B betacoronaviruses. Nat Microbiol. https://doi.org/10.1038/ s41564-020-0688

5. Istituto Superiore di Sanita: Report of characteristics of patients died positive for COVID-19 in Italy. https://www.epicentro.iss. it/coronavirus/bollettino/Report-COVID-2019_17_marzo-v2. pdf Article in Italian, last accessed on March 19th, 2020

6. ARNO Diabetes Observatory: Healthcare profile of the Italian diabetic population. https://www.siditalia.it/clinica/linee-guida -societari/send/80-linee-guida-documenti-societari/5025-rappo rto-arno-diabete-2019. Accessed 19 Mar 2019 
7. Zoppini G, Fedeli U, Schievano E, Dauriz M, Targher G, Bonora E, Corti MC (2018) Mortality from infectious diseases in diabetes. Nutr Metab Cardiovasc Dis 28:444-450
Publisher's Note Springer Nature remains neutral with regard to jurisdictional claims in published maps and institutional affiliations. 